\title{
Requirement of hollow process challenge device for monitoring hollow and complex instruments sterilization: a simulator for proper sterility assurance
}

\author{
Debabrata Basu MSc, DHM ${ }^{1}$ \\ Tata Medical Center, Kolkata, India
}

To the Editor-The central sterile supply department (CSSD) acts as a backbone of any hospital services in which the sterilization of a wide range of materials is required for therapeutic, diagnostic, and infection control purposes. Depending upon the nature of materials, these items are sterilized using various sterilization processes such as steam sterilization, ethylene oxide sterilization, and hydrogen peroxide sterilization. The CSSD is committed to maintain a high level of standard in respect of quality assurance and quality control activities. To ensure the quality of equipment performance, several parameters are used to monitor the effectiveness of sterilization processes, including physical, chemical, and biological parameters. The overall vision is to ensure quality in all deliverables, not just sterile supplies. ${ }^{1,2}$

The CSSD supplies various sterile materials (eg, surgical instruments, reusable linens, and dressing materials) to clinical areas for patient care purposes. According to the Spaulding classification, most surgical instruments that touch blood and sterile tissues are categorically critical. ${ }^{3}$ Designwise, surgical instruments are of 2 types: solid and hollow. The sterilization of solid instruments is simple because the sterilant (eg, steam) can easily reach solid surfaces to destroy microorganisms. In contrast, the sterilization of hollow instruments (eg, suction tube or catheters) is difficult because air is entrapped easily inside the luminal instruments blocks steam from reaching all of the inner surfaces. Thus, for luminal instruments, satisfactory sterilization is not possible and the medical devices remain unsterile.

These problems can be overcome in 2 ways: inoculation test for culture or simulation test by hollow-process challenge device (HPCD). Both methods can be used to monitor the sterility of hollow and complex instruments.

The inoculation test is carried out by direct inoculation of complex hollow instruments at the most difficult penetration location, where usual biological indicators cannot reach. The inoculation test is the only way to check sterilization inside complex instruments and sealed areas. To check the efficacy of the sterilization procedure, the correct inoculation with a defined population is required at the worst-case location. For the steam process, suspension of $G$. stearothermophilus with a $40 \%$ ethanol concentration is required. This test cannot be used for routine monitoring, but it can be used for validation (EN ISO 17665-1, ISO 14937) or sterility

Author for correspondence: Debabrata Basu, MSc, DHM, Tata Medical Center, 14 Major Arterial Road (E-W), New Town, Rajarhat, Kolkata 700 160, India. Email: debabrata.basu@ tmckolkata.com

Cite this article: Basu D. (2019). Requirement of hollow process challenge device for monitoring hollow and complex instruments sterilization: a simulator for proper sterility assurance. Infection Control \& Hospital Epidemiology, 40: 951-952, https://doi.org/ $10.1017 /$ ice. 2019.138 testing purposes. For routine monitoring, HPCD testing (per EN867-5) is the better alternative because it can accurately simulate the luminal instruments by measuring their inner surfaces. ${ }^{4}$

The HPCD determines the hollow penetration resistance of a sterilizer, and a few HPCDs are required with different lengths and diameters, but wall thickness (eg, $0.5 \mathrm{~mm}$ ) and tube materials (ie, polytetrafluoroethylene or stainless steel) are the same. The tube is open on one side for steam penetration and the other side is closed, with a capsule containing a biological indicator (per EN ISO 111381) or chemical indicator (per ISO 11140-1). The HPCDs are placed in the most challenging areas of the sterilizer (eg, in front of the door or above the drain strainer) in the worst-case scenario. After completion of the sterilization cycle, the results reveal that some of the chemical or biological indictor pass and others fail. The maximum size (inner diameter $\times$ inner length) that passes becomes the benchmark of the penetration capacity of the sterilizer. ${ }^{5}$

Worldwide, there are 2 types of steam sterilizers: the gravity type and the prevacuum type. The prevacuum sterilizer is more efficient than the gravity type with respect to the air removal process. Sterilization of solid instruments is easy (ie, only surface sterilization) because they can be sterilized using both gravity and prevacuum sterilization systems and because they can be monitored using any conventional method (ie, normal biological or chemical monitoring). Conversely, the sterilization of complex instruments is critical and can only be performed with a vacuum-assisted sterilization system due to the possibility of air pocket formation inside the luminal instruments. Under such conditions, no steam condenses and the sterilization process becomes suboptimal. Thus, for complex hollow instruments, HPCD is the only option for correct evaluation. ${ }^{6}$

Today, more and more infectious diseases pose serious threats to human health. A patient can be infected before admission to the hospital (ie, community-acquired infection or CAI) or may be infected after hospital admission (ie, hospital-acquired infection or HAI). Many HAIs are associated with prolonged hospital stays: ventilator-associated pneumonia (VAP), catheter-associated urinary tract infection (CAUTI), central-line-associated bloodstream infection (CLABSI), surgical-site infection (SSI), and so on. Although CSSD services are basically responsible for preventing SSI (eg, laparoscopic port infection, infection from implant procedure), which is an element of HAI. ${ }^{7,8}$ However, minimizing SSI also depends on various factors, and sterilization monitoring is an important aspect of in these prevention efforts. The main difficulty in India is to the use of costly disposable medical devices (eg, cardiac catheter, Octopus, suction tube, robotic and laparoscopic instruments, dental and ophthalmic instruments), which drives up the procedure cost. ${ }^{9}$ Reprocessing medical devices and proper monitoring are required to minimize the risk of infection. To ensure sterility, a proper simulator that can validate the entire 
sterilization process is recommended. For monitoring proper steam penetration inside hollow or complex instruments (ie, luminal instruments), HPCD is clearly the best option. ${ }^{10}$

\section{References}

1. Basu D, Bhattacharya S, Mahajan A, Ramanan VR, Chandy M. The importance of the central sterile supply department in infection prevention and control. Infect Control Hosp Epidemiol 2014;35:1312-1314.

2. Basu D, Bhattacharya S, Mahajan A, Ramanan VR, Chandy M. Sterilization indicators in central sterile supply department: quality assurance and cost implications. Infect Control Hosp Epidemiol 2015;36:484-486.

3. McDonnell G, Burke P. Disinfection: is it time to reconsider Spaulding? J Hosp Infect 2011;78:163-170.

4. Steri-record direct inoculation syringe to test sterility inside complex instruments. gke website. https://www.gke.eu/en/product-cataloguesand-data-sheets.html?file=files/gke_2013/content/en/downloads/Data\% 20sheets/Biological\%20indicators/Direct\%20Inoculation\%20Syringe.pdf. Published 2013. Accessed April 18, 2019.

5. Current reference devices for hollow instruments loads as defined in standards are not a valid steam penetration test. gke website. http://www.gke.de/en/publications.html?file=files/gke_2013/content/en/ downloads/Publications/Others/Current\%20reference\%20devices\%20for \%20hollow\%20instruments\%20loads.pdf. Published 2013. Accessed May 11, 2018.

6. Effectiveness of hospital central sterilization processing vs clinic-based sterilization protocols: a noninferiority trial. Dalhousie University Library website. https://dalspace.library.dal.ca/bitstream/handle/10222/ 54542/Stefanuto-Peter-MSc-OMFS-September-2014.pdf?sequence $=5$. Published 2014. Accessed May 11, 2018.

7. Lobdell KW, Stamou S, Sanchez JA. Hospital-acquired infections. Surg Clin N Am 2012;92:65-77.

8. Sasmal PK, Mishra TS, Rath S, Meher S, Mohapatra D. Port site infection in laparoscopic surgery: a review of its management. World J Clin Cases 2015;3:864-871.

9. Cost comparison of medical treatments: India vs the world. India Profile Medical Tourism website. http://www.indiaprofile.com/medical-tourism/ cost-comparison.html. Accessed April 10, 2018.

10. Performance of hollow load process challenge devices (HLPCDs) for the determination of air removal and steam penetration in porous load steam sterilization processes. University of Glascow Enlighten website. http://eprints.gla.ac.uk/136299/1/136299.pdf. Accessed May 10, 2018.

\title{
Serotype 14 pneumococcal bacteremia: From one neonate to another in a pediatric intensive care unit
}

\author{
Audrey Vincent $\mathrm{MD}^{1}$, Stéphane Bonacorsi MD, $\mathrm{PhD}^{2,3}$, Emmanuelle Varon $\mathrm{MD}^{4}$, Stéphane Dauger MD, $\mathrm{PhD}^{1,3}$ and \\ Michael Levy MD, PhD ${ }^{1,3}$ (i) \\ ${ }^{1}$ Pediatric Intensive Care Unit, Robert-Debré University Hospital, Assistance Publique Hôpitaux de Paris, Paris, France, ${ }^{2}$ Microbiology Unit, Robert-Debré \\ University Hospital, Assistance Publique Hôpitaux de Paris, Paris, France, ${ }^{3}$ University Paris Diderot Sorbone Paris Cité, Paris, France and ${ }^{4}$ National Reference \\ Centre for Pneumococci, Centre Hospitalier Intercommunal, Créteil, France
}

To the Editor-Streptococcus pneumoniae is a leading cause of community-acquired bacterial infections, but an unusual source of neonatal sepsis ${ }^{1}$ and nosocomial cases have rarely been described. We report 2 original cases of neonatal S. pneumoniae infection due to the same serotype 14 pneumococcal strain that occurred in the same pediatric intensive care unit (PICU) with a highly probable nosocomial contamination from one to the other.

Patient 1 was a 13-day-old boy admitted to the PICU for respiratory distress due to respiratory syncytial virus (RSV)-induced bronchiolitis on November 11. He developed apnea with bradycardia and a deep decrease in blood oxygen requiring noninvasive ventilation using bilevel positive airway pressure. His respiratory distress worsened 24 hours after admission due to left pulmonary pneumoniae, and a blood culture was positive with a serotype 14 S. pneumoniae isolate with decreased susceptibility to penicillin G (Table 1) and resistance to macrolides and cotrimoxazole. The patient was treated with cefotaxime $(100 \mathrm{mg} / \mathrm{kg} /$ day $)$. Evolution was favorable with spontaneous ventilation on day 4 , and he was discharged from the PICU on day 5 (November 15).

Author for correspondence: Michael Levy, Pediatric Intensive Care Unit, Robert-Debré University Hospital, 48, boulevard Sérurier, 75019 Paris, France. Email: Michael.levy@aphp.fr Cite this article: Vincent A, et al. (2019). Serotype 14 pneumococcal bacteremia: From one neonate to another in a pediatric intensive care unit. Infection Control \& Hospital Epidemiology, 40: 952-953, https://doi.org/10.1017/ice.2019.152
Patient 2 was a 18-day-old healthy full-term boy also admitted to the PICU for respiratory distress due to RSV-induced bronchiolitis on November 9. He had a right superior lobe pulmonary atelectasis, no signs of bacterial infection, and negative blood culture. Noninvasive ventilation using continuous positive airway pressure was performed, followed 2 days later by invasive ventilation because of the onset of acute respiratory distress syndrome (ARDS). On November 15, his clinical state worsened, with bilateral pneumonia, and a tracheal sample yielded $S$. pneumoniae with the same pattern of antibiotic susceptibility as case 1. Central line insertion, prone positioning, high-frequency ventilation, nitric oxide, corticotherapy, and antibiotic treatment with cefotaxime $(100 \mathrm{mg} / \mathrm{kg} /$ day for 5 days) were performed. Fever stopped 2 days after the initiation of antibiotherapy, and the patient was removed from mechanical ventilation on November 22. Fever reappeared on November 24 due to central-line-associated bloodstream infection (CLABSI). Streptococcus pneumoniae was detected in qualitative peripheral and catheter blood cultures performed November 25, as well as in a quantitative catheter central-line blood culture with $10^{4}$ colony-forming units $(\mathrm{CFU}) / \mathrm{mL}$. The catheter culture after removal was positive, with $10^{6} \mathrm{CFU} / \mathrm{mL}$. This serotype 14 $S$. pneumoniae harbored the same profile of susceptibility to antibiotics as case 1 (Table 1). Cefotaxime $(150 \mathrm{mg} / \mathrm{kg} /$ day) was introduced and continued for a total duration of 7 days after the first sterile blood culture. The patient's fever disappeared, and he was discharged from the PICU on November 27. Considering the antibiotic susceptibility 